was $1.4 \mathrm{mg}$ (range $0-3.5$ ). 2 patients had local anaesthetic spray as an alternative.

PEG tube was successfully placed in 25 (96\%) patients; in 1 the procedure had to be abandoned due to laryngospasm and hypoxia. Median observed follow up post-PEG insertion was 186 days (range 16-677). There was 1 death within 30 days of PEG placement, at day 16 due to pneumonia superimposed on type 2 respiratory failure. 19 patients died (73\%) during follow up, all due to complications of the index disease, with median time to death 150 days (range 16-441). There were minor complications in 3 patients (12\%) (2 PEG site infection treated successfully, 1 respiratory depression requiring flumazenil).

Conclusion PEG placement can be safely and effectively achieved in MND patients with impaired respiratory function using non invasive ventilatory support. This offers a viable alternative to radiological or surgical techniques in these patients. We advocate a referral service for this specialised multi-specialty approach.

Disclosure of Interest None Declared.

\section{OC-037 THE INCREASING ROLE OF ENHANCED SEDATION ASSISTED ERCP: IMPORTANT LESSONS FOR SERVICE PROVISION}

D Joshi*, B Paranandi, G El Sayed, G Johnson, MH Chapman, SP Pereira, GJ Webster. Department of Gastroenterology, University College London Hospital, London, UK

\subsection{6/gutinl-2014-307263.37}

Introduction ERCP in the UK has historically been performed under conscious sedation (SED). However, given the increasing complexity of cases the role of enhanced sedation assisted ERCP (ENS ERCP) is increasing. A previous audit iat UCLH showed that intolerance of SED was a major factor in ERCP failure. BSG guidance was issued in 2011 regarding the use of propofol sedation for ERCP in the UK. ${ }^{1}$ We describe our experience of ENS ERCP and highlight the importance of the regular availability of this service.

Methods Our prospective ERCP database was interrogated to include cases between Jan-Nov 2013. Two dedicated ENS ERCP lists run weekly at UCLH. Data collection included procedural information, patient demographics, ASA status, Cotton grade of difficulty (1-4), and endoscopic/anaesthetic complications. ENS ERCP was defined as the use of propofol +/- fentanyl without the need for intubation. ENS was administered by consultant anaesthetists. Data presented as median with range. Comparison was made between SED and ENS ERCP patients.

Results During the 10 month study period 629 ERCPs were performed in 532 patients (52\% male). 423 procedures were performed under SED and 139 under ENS. ENS patients were younger compared to SED patients $(54,9-88$ years vs. $66,20-$ 96 years, $\mathrm{p}<0.0001)$ but ASA grade $1-2$ status was similar between the two groups ( 84 vs. $78 \%, \mathrm{p}=\mathrm{NS}$ ). An increased number of Cotton grade 3-4 ERCPs were perfomed in the ENS group (64 vs. $34 \%, \mathrm{p}<0.0001$ ). Common indications for ENS included previously uncomfortable/failed procedure (30\%), biliary/pancreatic sphincter of Oddi manometry (24\%) and single operator cholangioscopy (20\%). Patient choice accounted for only $4 \%$ of cases. $59 \%$ of cases were tertiary referrals, $12 \%$ of which had failed previously. $77 \%$ of referrals were elective cases, $12 \%$ urgent day-case referrals and $11 \%$ urgent in-patients. ERCP was completed successfully in $95 \%$ of cases. Anaesthetic complications occurred in 3 cases all relating to over sedation requiring intubation. ERCP-related complications occurred in 5\% of cases. Where previous SED ERCP was unsuccessful due to patient intolerance, the procedure was completed in all cases using ENS. Conclusion To date ENS ERCP has predominately been used for previously failed/poorly tolerated procedures and Cotton Grade 3-4 ERCPs. ENS ERCP improves outcomes and is safe when delivered with anaesthetic support. It is likely to be increasingly requested by patients and referrers. Regular ENS provision should be offered by all endoscopy units offering ERCP, and the anaesthetic resource and funding implications will need to be pursued.

\section{REFERENCE}

1 Guidance for the use of propofol sedation for adult patients undergoing ERCP and other complex upper GI endoscopy procedures, April 2011. RCoA and BSG guidance

Disclosure of Interest None Declared.

\section{OC-038 EFFECTIVENESS OF A NURSE-LED ALCOHOL LIAISON TEAM IN REDUCING ADMISSIONS AT LANCASHIRE TEACHING HOSPITALS NHS FOUNDATION TRUST}

S Soteriadou*, E Dermody, K Allan, K Anderson, A Platt, V Balachandrakumar, A Sharma. Department of Gastroenterology, Lancashire Teaching Hospitals NHS Foundation Trust, Preston, UK

\subsection{6/gutjnl-2014-307263.38}

Introduction In 2010/11 alcohol related harm cost the NHS in Lancashire $£ 141.92$ million, with Preston having the highest rate of hospital admissions for alcohol related liver disease in the North West. At that time there was no alcohol liaison team within Lancashire Teaching Hospitals. In view of this, in April 2013, the Hospital Alcohol Liaison Service (HALS) was created providing a seven day service for both the Royal Preston Hospital and Chorley and South Ribble Hospital.

Methods The HALS team comprises 4 senior nurses with experience in managing patients with alcohol and substance misuse. The referral criteria are patients scoring 8 or more on the Alcohol Use Disorders Identification Tool (AUDIT). A prospective database was created to include numbers of referrals, types of alcohol misuse, referring wards and departments, dates of admission and discharge, and the numbers of bed days saved. Data collected from April-October 2013 were analysed.

Results 808 patients were reviewed with $68 \%$ being male. The majority referrals were acute admissions, with $23 \%$ referred from the Emergency Department and 47\% from the Medical Assessment Unit. Patients were reviewed within an average of $12 \mathrm{~h}$ since referral time (range 3-36 h). Delayed discharges were frequently identified in patients on a reducing regime of Chlordiazepoxide. The majority of patients were being kept in to complete this regime, regardless of whether they planned to stop drinking or not. On discharge, patients were not being offered follow up in the community which often led to recidivism and re-attendance at hospital seeking further detoxification. The HALS team reviewed and assessed these patients with validated assessment tools including the Severity of Alcohol Dependency Questionnaire (SADQ) and Clinical Institute Withdrawal Assessment Score (CIWA). The level of misuse was calculated as low risk in 127 patients, dependent in 382, harmful in 126, hazardous in 166, detox in 1 and unknown in 6 patients. Existing treatment regimes were reviewed to ensure they were appropriate and timely, 
and patients were identified for safe and early discharge with early community follow up. A total of 641 bed days were saved over the time period analysed.

Conclusion Implementation of a 7-day HALS team covering two acute hospital sites has significantly improved the quality of care provided to patients with alcohol-related disorders. In addition there has been a very positive impact on reducing length of stay and bed occupancy. Plans are in place to develop and audit the HALS team further and strengthen working relationships with community partners.

Disclosure of Interest None Declared.

\section{OC-039 IMPROVING QUALITY IN A NATIONAL INTESTINAL FAILURE UNIT: GREATER EFFICIENCY, IMPROVED ACCESS, REDUCED MORTALITY}

${ }^{1} \mathrm{E}$ Donaldson*, ${ }^{2} \mathrm{M}$ Taylor, ${ }^{2} \mathrm{~A}$ Abraham, ${ }^{2} \mathrm{G}$ Carlson, ${ }^{2} \mathrm{O}$ Fletcher, ${ }^{2} \mathrm{~V}$ Jacqui, ${ }^{2} \mathrm{~A}$ Teubner, ${ }^{2} S$ Lal. 'Gastroenterology/Quality Improvement, Salford Royal NHS Foundation Trust, Salford, UK; ${ }^{2}$ Intestinal Failure Unit, Salford Royal NHS Foundation Trust, Salford, UK

\subsection{6/gutjnl-2014-307263.39}

Introduction In 2010, there was a significant waiting list for admission to the Intestinal Failure Unit (IFU) at Salford, one of two current nationally-accredited centres. There had also been a steady increase in referrals to the IFU (89 patients in 2005; 152 patients in 2012) and the number of established patients requiring home parenteral nutrition (HPN) (135 patients in 2005; 206 patients in 2012). The impact of the long waiting list for these complex patients was that patient deaths occurred in those awaiting admission. Furthermore, the 'Strategic Framework for IF and HPN Services' in England had earlier highlighted the need for services to 'foster equity of access'. ${ }^{1}$ The SRFT IFU team therefore conducted a rigorous assessment of its processes in order to improve patient flow and access to the IFU. The primary aim was to reduce inpatient length of stay (LOS) by $10 \%$.

Methods We employed continuous improvement methodology, utilising the Model for Improvement and running sequential Plan-Do-Study-Act cycles. In addition to the key flow data, such as LOS and referral times, process data were collected, including time to intravenous feeding line insertion, time to radiology studies etc., when looking to streamline specific areas of practice. Data were analysed using statistical process control charts produced using QI Macros (KnowWare International, INC.). Statistically significant shifts were determined 'a priori' according to standard operating principals for special cause variation. ${ }^{2}$

Results Process improvements yielded a $20.8 \%$ reduction in average length of stay on the IFU from 55.7 to 44.1 days and a reduction of $70.7 \%$ in the average length of time spent on the waiting list for admission from 65.1 to 19.1 days. These changes were associated with concomitant reduction in 30-day readmission rates from 12.1 to $4.5 \%$ and early suggestions of reduced waiting list mortality. The number of inpatient deaths did not increase; indeed, there was a sustained increase in the number of complete discharge episodes between inpatient deaths (mean increase from 13 to 44).

Conclusion A quality improvement model is an effective means of enhancing the efficiency of a large National centre dealing with complex medical and surgical patients. Improvements in inpatient efficiency can reduce waiting times for admission, thus improving access and reducing waiting list mortality. The improvements in efficiency can be achieved without compromising patient safety.

\section{REFERENCES}

1 Strategic Framework for Intestinal Failure and Home Parenteral Nutrition Services for Adults in England 2008

2 Langley, G et al. 2009. The improvement guide: a practical approach to enhancing organizational performance. John Wiley \& Sons

Disclosure of Interest None Declared.

\section{OC-040 COMMUNITY-BASED ENDOSCOPY IN A CAR PARK. FANTASY OR REALITY?}

A Hitchen, B Kompo*, P Phillips, E Price, G Deans, NK Ahluwalia. Gastroenterology, Care UK, Manchester, UK

\subsection{6/gutjnl-2014-307263.40}

Introduction Owing to safety and quality concerns raised by BSG in 1990s, Community Based Endoscopy (CBE) rapidly declined. In $2014 \mathrm{CBE}$ is back on the agenda with CCGs demanding a safe, high-quality, sub-tariff, 7 day endoscopy service close to the patient's home, at a time of the patient's choosing. We describe 5 years experience of a fully JAG accredited consultant-delivered completely mobile endoscopy service provided in shopping centre car parks throughout Greater Manchester (GM).

Methods In 2007, concept emerged out of a tender from 10 PCTs demanding safe and high quality endoscopy in the community. A linked-3-trailer unit with spacious waiting area for patients/relatives, consent-counselling, preparation, examination, decontamination and recovery areas. with its own water, electricity, waste disposal, e-communications and administration network was commissioned with integral office, staff kitchen/rest room and changing area. All staff undergo rigorous mandatory induction and regular updates, $\mathrm{CPD}$, audits as per JAG. Emergency scenarios are regularly rehearsed and audited. Full complement of ALS certified staff support consultants 8 am-8 pm, 7/7 [360] days in diagnostic UGI and LGI endoscopies [including polypectomies] and deliver 36 units/day. All patients undergo JAG-standard monitoring of pulse, $\mathrm{BP}, \mathrm{O}_{2}$ saturation, sedation and pain scores. All records are paperless, live and e-MAXIM andUNISOFT-based. All patients are contacted within $24 \mathrm{~h}$ by an experienced nurse to record any untoward incident. Patient and family feedback and regular meetings with GP allows total quality management in service delivery. Though no age limit, patients with IDDM, BMI $>40,>25$ st and ASA $\geq 3+$ are excluded. Quarterly audits are shared with commissioners. Unit relocates biweekly to 7 convenient locations with adjacent free parking.

Results Of 26599 (10539 UGI, 10583 Flexi-Sig, 5477 Colonoscopy) procedures, 1 in 3 patients opted for out-of-hours or weekend as first choice.

LGI- 93\% good-bowel prep, 91\% caecal intubation, 8.8\% adenoma detection, $97 \%$ polyp recovery and $100 \%$ Bx for diarrhoea. UGI- 98\% D2 intubation, 100\% 6 week repeat for GU, $84 \%$ unsedated. Biannual JAG-GRS compliant audits showed 0\% 30 day mortality, 0\% UGI SAE and $5(0.018 \%)$ unplanned hospital admissions 4 requiring surgery due to colon perforation [one post-flexi detected in the unit (diverticular disease), 1 from polypectomy, 2 diagnostic colonoscopy] and fifth due to hyponatremia (CitraFleet - now discontinued) requiring electrolyte correction.

Conclusion CBE is ready for prime time, just at the right time, as commissioners are now seeking more care in the community 